\title{
A Study on the Bi-Rayleigh ROC Curve Model
}

\author{
Sudesh Pundir and R. Amala
}

\begin{abstract}
Receiver Operating Characteristic (ROC) curves are used to describe and compare the accuracy of diagnostic test or the ability of a continuous biomarker in discriminating between the subjects into healthy or diseased cases in medical field. The most familiar form of ROC curve is Bi-normal (Gaussian) ROC curve model, which assumes that the test scores or a monotone transformation of the test scores are from two normal populations (i.e. healthy and diseased). It may not be true all the time, it may violate the assumptions of normal distribution in some situations and also we cannot adopt the model as it is when the sample size is small. In this paper, we have proposed ROC curve model for Rayleigh distribution which can be used even when sample size is small. The properties of Bi-Rayleigh ROC model are studied and Area Under the ROC Curve (AUC) are derived. The proposed model is supported by real life example as well as simulation studies. The confidence interval for the population parameter is studied with simulation studies of varying sample sizes. It is found that Bi-Rayleigh ROC model provides better accuracy of classification than the conventional bi-normal ROC model.
\end{abstract}

Keywords--- AUC, Bi-Rayleigh Distribution, Confidence Interval, ROC Model

\section{INTRODUCTION}

$\mathrm{I}^{\mathrm{N}}$ $\mathrm{N}$ recent years, the Receiver Operating Characteristic (ROC) curve has become one of the most powerful statistical techniques for classifying the subjects into one of the two populations in medical field [1]. It is also used to describe the accuracy of a diagnostic test and at the same time it can be used for comparing two diagnostic tests [2]. In classifying an individual, a biomarker (gold standard) of a particular test procedure is essential. If the measuring quantity exceeds the gold standard value, then that individual is classified as diseased otherwise healthy.

The most popular parametric model for an ROC curve is the Bi-normal model which assumes that the test scores in the two populations (i.e. diseased and healthy) are normally distributed. In medical diagnosis, plenty of research has been made on ROC curves by assuming that the test scores are Normal. But there are numerous situations where the test scores are non-normal. The test scores may follow some specific distribution (say) Rayleigh distribution in which case one can directly use the Bi-Rayleigh model without going for any sort of transformation in order to make the test scores normal. Here we have proposed another parametric model

Sudesh Pundir, Assistant Professor, Department of statistics, Pondicherry University, Puducherry, India. E-mail: sudeshpundir19@gmail.com

Amala R, Student, Department of statistics, Pondicherry University, Puducherry, India.E-mail: amalar.statistics@gmail.com called Bi-Rayleigh model which can be applied in the situations when the other parametric models viz. Bi-Normal, Bi-Exponential, Bi-Gamma and Bi-lomax models fails.

The Rayleigh distribution is named after Lord Rayleigh. It is a special case of Weibull distribution. As normal distribution, it has been widely used in various fields. In this paper we have applied the Rayleigh distribution in medical diagnostics. This paper is divided in the following sections.

In the ROC model section, Bi-Rayleigh ROC model and its AUC are derived and its properties are discussed. In application section, two real life example viz. Head trauma data and Multiple Sclerosis data were used for the proposed model and then these are compared with the conventional binormal model. In the simulation section, simulation studies have been carried out for different values of parameters to support the Bi-Rayleigh ROC model. In the last section, the confidence intervals for the parameter $\sigma_{i}, i=0,1$ are discussed for real life example and simulated data sets.

\section{ROC MODEL AND AREA UNDER THE CURVE}

Let the scores $(\mathrm{S})$ be a random variable in healthy $(\mathrm{H})$ and diseased (D) populations respectively. The ROC curve is plotted over values of threshold value ( $\mathrm{t}$ ) and is given by

$\mathrm{y}(\mathrm{t})=\mathrm{h}(\mathrm{x}(\mathrm{t}))$

where $\mathrm{x}(\mathrm{t})$ is the False positive rate which is given by

$$
\mathrm{x}(\mathrm{t})=\mathrm{P}(\mathrm{S}>\mathrm{t} \mid \mathrm{H})
$$

and $y(t)$ is True positive rate which is given by

$y(t)=P(S>t \mid D)$

The general functional form of ROC can be represented as

$$
Y=1-G\left[F^{-1}(1-x)\right], 0 \leq x \leq 1
$$

where $\mathrm{F}$ is the distribution function for healthy population and $\mathrm{G}$ is the distribution function for diseased population.

Let us assume that the distribution of the scores $\mathrm{S}$ from both the populations (healthy and diseased) follow Rayleigh distribution with single parameter $\sigma$. The probability density function of Rayleigh distribution is given by

$$
f(x)=\frac{x}{\sigma^{2}} e^{-\frac{x^{2}}{2 \sigma^{2}}}, x>0, \sigma>0
$$

and the cumulative distribution function is given by

$$
F(x)=1-e^{\frac{-x^{2}}{2 \sigma^{2}}}, x>0, \sigma>0
$$

Let $S_{i}, i=0,1$ be the scores, ' 0 ' denotes the test scores from healthy population and ' 1 ' denotes the test scores are from 
diseased population. Let $\mathrm{X}_{11}, \mathrm{X}_{12}, \mathrm{X}_{13}, \ldots \ldots \ldots \mathrm{X}_{1 \mathrm{n} 1} \sim$ Rayleigh $\left(\sigma_{1}\right)$ and $\mathrm{X}_{01}, \mathrm{X}_{02}, \mathrm{X}_{03}, \ldots \ldots \ldots \mathrm{X}_{0 \mathrm{n} 0} \sim$ Rayleigh $\left(\sigma_{0}\right)$ having the density function as

$$
f(x)=\frac{x_{i}}{\sigma_{i}^{2}} e^{-\frac{x_{i}^{2}}{2 \sigma_{i}^{2}}}, x_{i}>0, \sigma_{i}>0, \mathrm{i}=0,1
$$

with $m_{0}=\sigma_{0} \sqrt{\frac{\pi}{2}} \cong 1.253 \sigma_{0}$ and $(4-\pi) \frac{\sigma_{0}^{2}}{2} \cong 0.429 \sigma_{0}^{2}$ are the mean and variance respectively for healthy population and $m_{1}=\sigma_{1} \sqrt{\frac{\pi}{2}} \cong 1.253 \sigma_{1} \quad \cong \quad 1.253 \sigma_{1} \quad$ and $(4-\pi) \frac{\sigma_{0}^{2}}{2} \cong 0.429 \sigma_{0}^{2}$ are the mean and variance respectively for diseased population. It is assumed that $\mathrm{m}_{1}>\mathrm{m}_{0}$.

The ROC model for bi-rayleigh distribution is obtained as

$$
\begin{aligned}
y(x(t))= & \left(\exp \left[-t^{2} / 2 \sigma_{0}^{2}\right]\right) \frac{\sigma_{0}^{2}}{\sigma_{1}^{2}} \\
& =\left(\exp \left[-t^{2} / 2 \sigma_{0}^{2}\right]\right)^{\gamma}
\end{aligned}
$$

where

$$
\gamma=\frac{\sigma_{0}^{2}}{\sigma_{1}^{2}}
$$

The form of the ROC curve changes as per the following three conditions.

i. If $\sigma_{1}^{2}=\sigma_{0}^{2}$, then the ROC curve agrees with the chance diagonal. In such a case the probability of allocating an individual to diseased population is same as that of allocating the individual to healthy population, so the TPR will always equal to FPR(i.e. a line joining $(0,0)$ and $(1,1))$

ii. If $\sigma_{1}^{2}>\sigma_{0}^{2}$, then the ROC curve lies above the chance diagonal showing some ability to discriminate between positive and negative cases. If $\sigma_{1}^{2}$ become more the curve tends to lie in upper-left corner of the unit square.

iii. If $\sigma_{1}^{2}<\sigma_{0}^{2}$, then the curve has no ability in discriminating the individuals into positive and negative group.

Some of the properties satisfied by the ROC curve for BiRayleigh model are given below

1. The Bi-Rayleigh ROC model $y(x(t))$ is a monotonic increasing function in the positive quadrant lying between $\mathrm{y}=0$ at $\mathrm{x}=0$ and $\mathrm{y}=1$ at $\mathrm{x}=1$.

2. The threshold value is well defined provided the slope of the ROC curve at the given point.

3. The Bi-Rayleigh ROC curve is unaltered if the classification scores undergo a strictly increasing transformation.

\subsection{Area Under the ROC Curve (AUC)}

It measures the accuracy of the test that tells us how well the test separates the group being tested into those with and without the disease. It is defined as $A U C=\int_{0}^{1} y(x) d x$

The higher is the value of AUC, better will be the performance of a test. Suppose, for a test, $\mathrm{AUC}=0.83$, it mean $83 \%$ accurate classification made using that diagnostic test.

The AUC expression for the Bi-Rayleigh distribution is given by

$$
A U C=\frac{\sigma_{1}^{2}}{\sigma_{1}^{2}+\sigma_{0}^{2}}
$$

Generally, the parametric values 0 and 1 of the two population under study are not known and their values are estimated from the sample. Let $\hat{\sigma}_{0}^{2}$ and $\hat{\sigma}_{1}^{2}$ are the estimates of parameter $\sigma_{0}^{2}$ and $\sigma_{1}^{2}$ respectively. So the estimated AUC is given as

$$
A U C=\frac{\hat{\sigma}_{1}^{2}}{\hat{\sigma}_{1}^{2}+\hat{\sigma}_{0}^{2}}
$$

\section{IMPLEMENTATION OF ROC MODEL ON REAL LIFE DATA}

In order to establish the Bi-Rayleigh ROC model and AUC, we have used two data sets in medical application. The two data sets are Multiple Sclerosis data and Head trauma data.

\subsection{Multiple Sclerosis Data}

Multiple Sclerosis (abbreviated as MS, known as disseminated sclerosis or encephalomyelitis disseminata) is an inflammatory disease in which the fatty myelin sheaths around the axons of the brain and spinal cord are damaged, leading to demyelination and scarring as well as a broad spectrum of signs and symptoms. Disease onset usually occurs in young adults and it is more common in women. MS was first described in 1868 by Jean-Martin Charcot.

MS affects the ability of nerve cells in the brain and spinal cord to communicate with each other effectively. Nerve cells communicate by sending electrical signals called action potentials down long fibers called axons, which are contained within an insulating substance called myelin. When myelin is lost, the axons can no longer effectively conduct signals. The name Multiple Sclerosis refers to scars (scleroses-better known as plaques or lesions) particularly in the white matter of the brain and spinal cord, which is mainly composed of myelin.

For detecting Multiple sclerosis one of the possible laboratory tests is the cerebrospinal fluid (CSF) immunoglobulin $\mathrm{G}(\mathrm{IgG})$ index. CSF immunoglobulin index is defined as the ratio of (IgG) in $\mathrm{CSF} / \mathrm{IgG}$ in Serum) to CSF- 
albumin/serum albumin. High values of the CSF IgG index are suspicious for multiple sclerosis. The data has been collected on 40 patients (Ref. table 2). Among them 20 were affected by other neurological disorders [1].

\subsection{Head Trauma Data}

For studying Bi-Rayleigh model we consider another data set. The test scores are the cerebrospinal fluid CK-BB (Creatine Kinase-BB) iso-enzyme measured within 24 hours of injury for predicting the outcome of severe head trauma. Cerebrospinal fluid (CSF) is a clear, colorless, bodily fluid that occupies the subarachnoid space and the ventricular system around and inside the brain and spinal cord. In essence, the brain "floats" in it.CSF acts as a cushion, protecting the brain and spine from injury. Here, CK-BB iso-enzyme measurements are considered to predict whether the patient has a good outcome or poor outcome.

A sample of 60 subjects admitted to a hospital with severe head trauma is considered (Ref. Table 1). Among them 19 of whom had a chance of full recovery and 41 of who had poor or no recovery. Higher values of CK-BB are suspicious to die or to get severe disability after suffering a severe head trauma [1].

Table 1: Head Trauma Data

\begin{tabular}{|l|l|c|}
\hline \multicolumn{2}{|c|}{ Poor outcome } & Good outcome \\
\hline 140 & 913 & 136 \\
\hline 1087 & 509 & 286 \\
\hline 230 & 576 & 281 \\
\hline 183 & 671 & 23 \\
\hline 1256 & 80 & 200 \\
\hline 700 & 490 & 146 \\
\hline 16 & 156 & 220 \\
\hline 800 & 356 & 96 \\
\hline 253 & 350 & 100 \\
\hline 740 & 323 & 60 \\
\hline 126 & 1560 & 17 \\
\hline 153 & 120 & 27 \\
\hline 283 & 216 & 126 \\
\hline 90 & 443 & 100 \\
\hline 303 & 523 & 253 \\
\hline 193 & 76 & 70 \\
\hline 76 & 303 & 40 \\
\hline 370 & 353 & 6 \\
\hline 543 & 206 & 46 \\
\hline
\end{tabular}

Table 2: Multiple Sclerosis Data

\begin{tabular}{|l|l|l|l|}
\hline \multicolumn{2}{|c|}{ Actually Positive } & \multicolumn{2}{c|}{ Actually Negative } \\
\hline 0.86 & 0.46 & 0.86 & 0.66 \\
\hline 3.23 & 2.49 & 0.88 & 0.41 \\
\hline 1.36 & 0.64 & 1.04 & 0.17 \\
\hline 1.02 & 2.04 & 1.17 & 0.64 \\
\hline 2.06 & 1.01 & 0.69 & 0.4 \\
\hline 2.17 & 1.64 & 0.4 & 0.85 \\
\hline 0.44 & 0.9 & 0.49 & 0.8 \\
\hline 2.73 & 2.89 & 1.11 & 0.68 \\
\hline 2.16 & 1.95 & 0.61 & 0.45 \\
\hline 0.52 & 2.74 & 0.4 & 0.15 \\
\hline
\end{tabular}

The fitness of these two data sets has been tested using Kolmogorov-smirnov test, Anderson-Darling test and Chisquare goodness of fit test by the software Easy Fit and the details has furnished below. The $\mathrm{p}$-values/statistic value corresponding to all the tests for Rayleigh distribution has been given in table 3 .

Table 3: Goodness of fit test

\begin{tabular}{|c|c|c|c|c|}
\hline \multirow[b]{3}{*}{$\begin{array}{l}\text { Name of the } \\
\text { Test }\end{array}$} & \multicolumn{4}{|c|}{ P-Value/Statistics } \\
\hline & \multicolumn{2}{|c|}{ Head trauma data } & \multicolumn{2}{|c|}{ M S data } \\
\hline & $\begin{array}{l}\text { Good } \\
\text { Outcome }\end{array}$ & $\begin{array}{l}\text { PoorOutc } \\
\text { omes }\end{array}$ & Positive & Negative \\
\hline $\begin{array}{l}\text { Kolmogorov } \\
\text { Smirnov }\end{array}$ & 0.36751 & 0.04414 & 0.63415 & 0.61261 \\
\hline $\begin{array}{l}\text { Anderson- } \\
\text { Darling } \\
\end{array}$ & 2.251 & 4.6579 & 0.53178 & 0.35707 \\
\hline Chi-Squared & 0.1645 & 0.01363 & 0.70235 & 0.99907 \\
\hline
\end{tabular}

The multiple Sclerosis data set fits the given distribution well in all the three goodness of fit test at alpha levels 0.2, 0.1, $0.05,0.02,0.01$. The good outcome of head trauma data is fitting the distribution for alpha level 0.02 and 0.01 for Kolmogorov-Smirnov test; 0.01 for Chi-Squared test. The good outcome of head trauma data is fitting the distribution for alpha level 0.2, 0.1, 0.05, 0.02 and 0.01 for KolmogorovSmirnov test; 0.05, 0.02, 0.01 for Anderson-Darling; 0.1, 0.05, 0.02 and 0.01 for Chi-Squared tests.

\subsection{Comparison with BI-Normal ROC Model}

Head Trauma data does not follow normal distribution but the transformed data that is after taking natural logarithm, it follows Normal distribution. After transforming the data we have plotted the bi-normal ROC curve. Its accuracy is only 0.8128. But for the same data without going for any transformation, it follows Rayleigh distribution. When we use Bi-Rayleigh ROC model the accuracy obtained is 0.9297 which is much better than bi-normal ROC model. It is also clear from the figure 1 where ROC curve for Bi-Rayleigh distribution dominates ROC curve for bi-normal ROC curve.

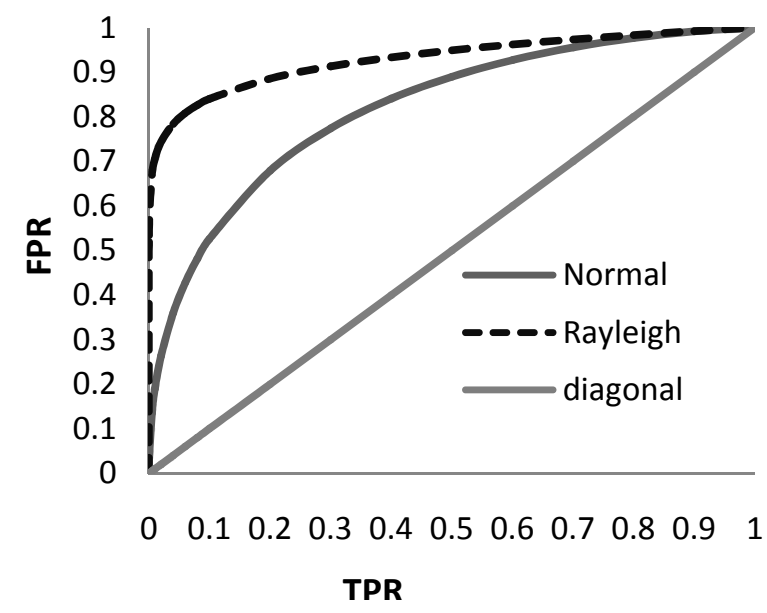

Figure1: Comparison of Rayleigh ROC Curve with Normal ROC Curve Using Head Trauma Data 
Similarly, MS data follows Rayleigh distribution well, as compared to normal distribution (Ref. section 3.2). BiRayleigh ROC model gives measure of accuracy as 0.87 which is more than the accuracy 0.81 obtained by using binormal ROC model. From Fig. 2 it is clear that, Bi-Rayleigh ROC curve dominates the bi-normal ROC curve for MS data.

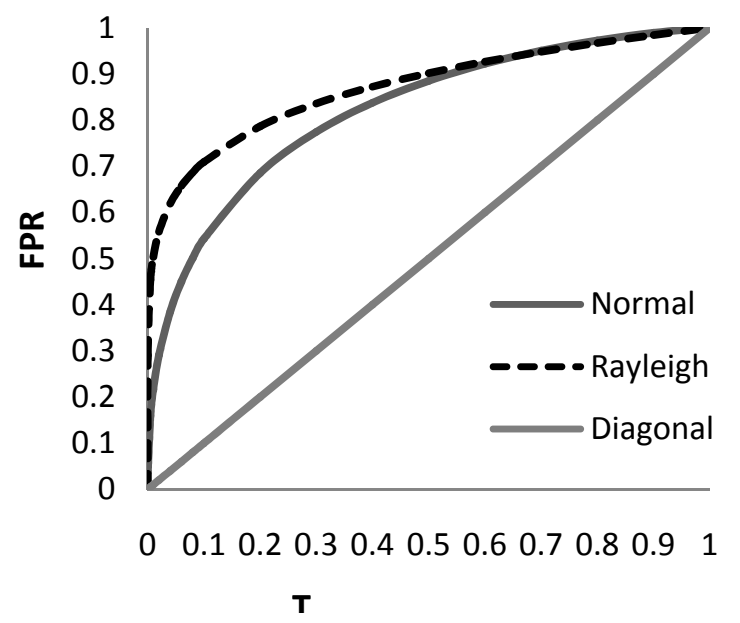

Figure 2: Comparison of Rayleigh ROC Curve with Normal ROC Curve using Multiple Sclerosis Data

One of the general approach in assessing the difference in the areas under two ROC curve derived from the same patients [5] is finding the $\mathrm{Z}$ ratio which is given by,

$$
z=\frac{A_{1}-A_{2}}{\sqrt{S E_{1}^{2}+S E_{2}^{2}-2 r S E_{1} S E_{2}}}
$$

where $\mathrm{A}_{1}$ and $\mathrm{SE}_{1}$ refers to the observed area and estimated standard error of the ROC area associated with the bi-normal model; and $\mathrm{A}_{2}$ and $\mathrm{SE}_{2}$ refers to the observed area and estimated standard error of the ROC area associated with the Bi-Rayleigh model; $r$ is the estimated correlation between $A_{1}$ and $A_{2}$. The $z$ value is then compared with the critical value then the decision has to be taken to accept or not to accept the hypothesis that no difference between two AUC's.

Here the standard errors are obtained by the variance of the Wilcoxon statistic [3]. Here we have two intermediate correlation coefficients. One is the correlation coefficient $\left(\mathrm{r}_{\mathrm{H}}\right)$ for the scores of healthy individuals for two models. Since we are using the same data set for both the models the correlation among them is 1 . Another is the correlation coefficient $\left(r_{D}\right)$ for the scores of diseased individuals for the two models. Obviously $r_{D}$ is also 1 . The correlation induced by the two areas $(r=0.92)$ are obtained from the table provided by [4] by using the average AUC for the two ROC curves and the average correlation coefficient $r_{D}$ and $r_{H}$. So the calculated $z$ ratio for head trauma data is 4.1424 which is greater than 1.96 can be taken as an evidence that the two areas are significantly different. By this argument we can say that Bi-Rayleigh ROC model is better than the normal ROC model in case of small samples. But for the MS data sets, $\mathrm{z}$ is $2.6086 \geq 1.96$, so we can interpret that there is a difference in two areas obtained by Rayleigh and normal models.

\subsection{Simulation Studies}

In general, to investigate the behavior of the ROC curve derived from the Bi-Rayleigh model, observations are generated from the Uniform distribution. Given a random variable $\mathrm{U}$ drawn from the Uniform distribution in the interval $(0,1)$, then the variate has a Rayleigh distribution with parameter $\sigma$.

$$
\mathrm{X}=\sigma \sqrt{-2 \ln (u)}
$$

The ROC curve of the Bi-Rayleigh model from generated data with sample size $\mathrm{n}_{1}=50$ from the diseased population and $\mathrm{n}_{0}=50$ from the healthy population with the parameter $\sigma_{1}=12$ and $\sigma_{0}=3$ respectively is given below.

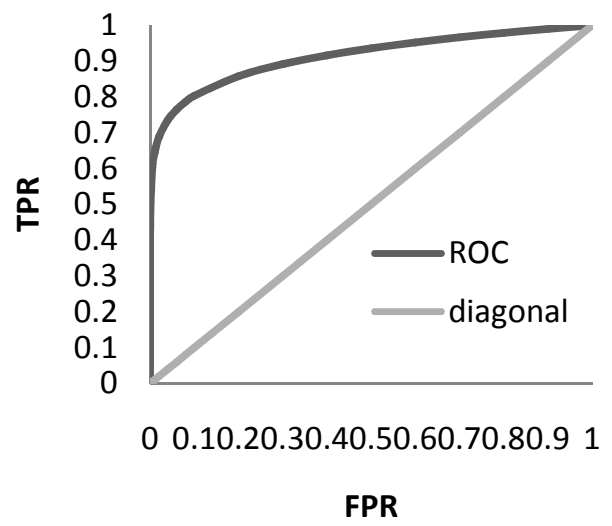

Figure 3: ROC Curve for Model with Parameter $\sigma 1=12$ and $\sigma 0=3$

The ROC curve plotted for bi-rayleigh model for different values of parameters $\sigma_{1}=12,10,8,6$ and fixed $\sigma_{0}=3$ is shown in Fig.4. It is concluded that for the fixed value of $\sigma_{0}$ and increasing value of $\sigma_{1}$, the ROC is more close to the upper right corner of the unit square that means one have higher degree of accuracy by looking at the AUC.

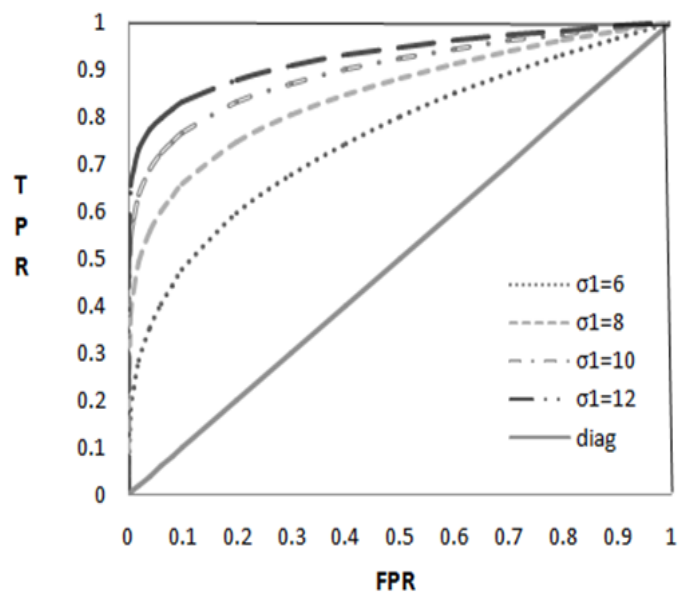

Figure 4: Roc Curve for Model with Parameter $\Sigma 1=12,10,8,6$ And $\Sigma 0=3$ 
When a sample of size 50 is taken for healthy and diseased population from Rayleigh distribution, the value of AUC for different values of the parameters $\sigma_{0}$ and $\sigma_{1}$ is provided in table 4.

Table 4: AUC for Different Values of $\sigma 0$ and $\sigma 1$

\begin{tabular}{|l|l|l|l|}
\hline \multirow{2}{*}{ S.No } & \multicolumn{2}{|l|}{ Value of $\sigma$} & \multirow{2}{*}{ AUC } \\
\cline { 2 - 3 } & $\sigma_{1}$ & $\sigma_{0}$ & \\
\hline 1 & 12 & 3 & 0.97144 \\
2 & 10 & 3 & 0.93795 \\
3 & 8 & 3 & 0.89478 \\
4 & 6 & 3 & 0.84478 \\
\hline
\end{tabular}

From Table 4, it is clear that for fixed value of $\sigma_{0}$ and as the value of $\sigma_{1}$ increases, the AUC increases.

The ROC curve for different values of parameters $\sigma_{0}=1,2$, 3 and fixed value of $\sigma_{1}=12$ is shown in Fig. 5. It is concluded that, as we increased the difference between $\sigma_{1}$ and $\sigma_{0}$, the ROC become closer to the upper left corner of the unit square that means one have higher degree of accuracy by looking at the AUC in Table 5.

Figure 5 shows that the ROC curve for Bi-Rayleigh model with fixed parameter for diseased viz. $\sigma_{1}=12$ and varying values of parameter for the healthy viz. $\sigma_{0}=1,2,3$.

Table 5: AUC for Different Values of $\sigma 0$ and Fixed $\sigma 1$

\begin{tabular}{|c|c|c|c|}
\hline \multirow[b]{2}{*}{ S.No } & \multicolumn{2}{|c|}{ Value of $\sigma$} & \multirow[b]{2}{*}{ AUC } \\
\hline & $\sigma_{1}$ & $\sigma_{0}$ & \\
\hline 1 & 12 & 1 & 0.991 \\
\hline 2 & 12 & 2 & 0.965362 \\
\hline 3 & 12 & 3 & 0.925299 \\
\hline
\end{tabular}

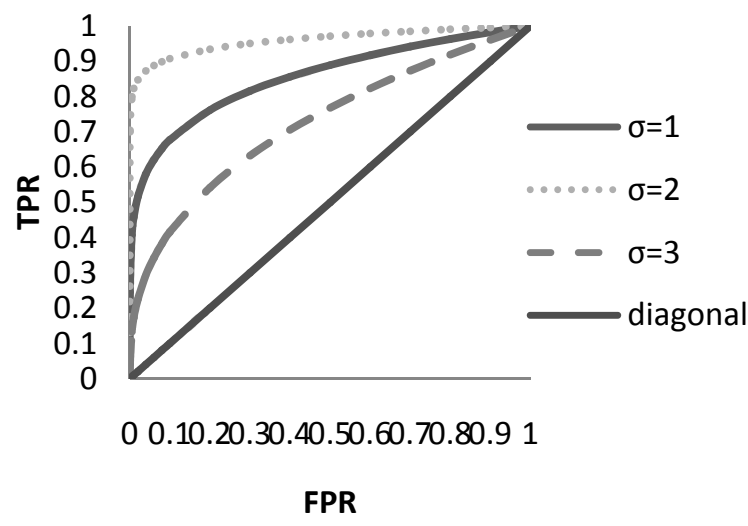

Figure 5: ROC Curve for Bi-Rayleigh Model with Parameters $\sigma_{1}=5$ and $\sigma_{0}=1,2,3$

It is clear that AUC decreases as $\sigma_{0}$ increases for fixed $\sigma_{1}$.

\subsection{Confidence Interval for $\sigma_{i}$ from Simulated Data}

Table 7 summarizes the confidence interval for $\sigma_{0}$ and $\sigma_{1}$ for different sample sizes using generated observation from Rayleigh distribution with parameter $\sigma_{1}=12 \sigma_{0}=4$.

\section{CONFIDENCE INTERVAL FOR POPULATION PARAMETER $\Sigma$ IN CASE OF LARGE SAMPLES}

By using Central limit theorem, we can find the confidence bound for the parameter $\sigma_{\mathrm{i}}$. The explicit form of confidence interval is $\left[\widehat{\sigma}_{l}-\operatorname{sd~} Z_{\alpha / 2}<i_{\mathrm{f}}<\widehat{\sigma}_{l}+\operatorname{sd} Z_{\alpha / 2}\right]$, where $\widehat{\sigma}_{l}$ is the estimator of the parameter $\sigma_{\mathrm{i}}$ and sd is the standard deviation of $\widehat{\sigma}_{l}, Z \alpha / 2$ is the tabulated value at the significance level $\alpha$.

From Table 6, we can see that even though the transformed data fits well for normal distribution, the accuracy is not worthy when compared to accuracy obtained by Bi-Rayleigh ROC model. From this, one can easily infer that when the test scores follow Rayleigh distribution it is worthwhile to use the Bi-Rayleigh ROC model instead of any other model.

Table 6: A Comparative Study Made for Head Trauma Data and Multiple Sclerosis Data for Rayleigh and Normal Distribution

\begin{tabular}{|c|c|c|c|}
\hline Data & Characteristics & Rayleigh & Normal \\
\hline \multirow{5}{*}{$\begin{array}{l}\text { Head } \\
\text { Trauma } \\
\text { data }\end{array}$} & AUC & 0.9297 & 0.81284 \\
\hline & \multirow[t]{2}{*}{ Estimates } & $\sigma_{1}=341.0157$ & $\mu_{1}=5.67$ \\
\hline & & $\sigma_{0}=93.7959$ & $\mu_{0}=4.37$ \\
\hline & \multirow{2}{*}{$\begin{array}{l}\text { Confidence } \\
\text { interval }\end{array}$} & $\begin{array}{l}{[226.954<} \\
\left.\sigma_{1}<455.077\right]\end{array}$ & $\begin{array}{l}5.35< \\
\left.\mu_{1}<5.99\right]\end{array}$ \\
\hline & & $\begin{array}{l}{[52.8266<} \\
\left.\sigma_{0}<134.765\right]\end{array}$ & $\begin{array}{l}3.938< \\
\left.\mu_{0}<4.805\right]\end{array}$ \\
\hline \multirow{5}{*}{$\begin{array}{l}\text { Multiple } \\
\text { Sclerosis } \\
\text { data }\end{array}$} & $\mathrm{AUC}$ & 0.8703 & 0.8112 \\
\hline & \multirow{2}{*}{ Estimates } & $\sigma_{1}=1.3292$ & $\mu_{1}=0.3342$ \\
\hline & & $\sigma_{0}=0.5132$ & $\mu_{0}=-0.5649$ \\
\hline & \multirow{2}{*}{$\begin{array}{l}\text { Confidence } \\
\text { interval }\end{array}$} & $\begin{array}{c}{[0.9361<} \\
\left.\sigma_{1}<1.7223\right]\end{array}$ & $\begin{array}{l}{[0.0486<} \\
\left.\mu_{1}<0.6199\right]\end{array}$ \\
\hline & & $\begin{array}{c}{[0.3865<} \\
\left.\sigma_{0}<0.6398\right]\end{array}$ & $\begin{array}{l}{[-0.8085<} \\
\left.\mu_{0}<-0.3213\right]\end{array}$ \\
\hline
\end{tabular}

Table 7: Confidence Interval for $\sigma_{i},, i=0,1$

\begin{tabular}{|c|c|c|c|c|c|c|}
\hline \multirow[t]{2}{*}{ S.No } & \multicolumn{2}{|c|}{$\begin{array}{l}\text { Sample } \\
\text { sizes }\end{array}$} & \multirow{2}{*}{$\widehat{\sigma_{1}}$} & \multirow{2}{*}{$\sigma_{1}$} & \multirow[t]{2}{*}{$\widehat{\sigma_{0}}$} & \multirow{2}{*}{$\sigma_{0}$} \\
\hline & $\mathrm{n}_{1}$ & $\mathrm{n}_{0}$ & & & & \\
\hline \multirow{7}{*}{5} & \multirow{7}{*}{$\begin{array}{l}100 \\
100 \\
200 \\
300 \\
400\end{array}$} & \multirow{2}{*}{50} & \multirow{3}{*}{11.5888} & $\begin{array}{l}{[10.5068,} \\
12.6696]\end{array}$ & \multirow{3}{*}{$\begin{array}{c}4.19921 \\
3.884\end{array}$} & $\begin{array}{l}{[3.6899,} \\
4.7084]\end{array}$ \\
\hline & & & & {$[128777$} & & 535028 \\
\hline & & 100 & & 12.8903] & & $4.27]$ \\
\hline & & 100 & 11.4813 & [10.3821, & 4.0977 & [3.59115, \\
\hline & & 200 & 12.36063 & {$[11.4678$,} & & 3.499 \\
\hline & & 300 & 11.65943 & 13.2534] & 3.8864 & 4.2736] \\
\hline & & & & $\begin{array}{l}{[10.7677,} \\
12.5512]\end{array}$ & 4.0103 & $\begin{array}{l}{[3.6289,} \\
4.2699]\end{array}$ \\
\hline
\end{tabular}

\section{CONCLUSION}

Zmedical diagnostic literature, it is always assumed that the test scores follow normal distribution even when the sample size is small and without checking for assumptions of 
the other distributions. In this paper, we have proposed an alternative ROC model which can be used when the normality assumption is not met. ROC model and the AUC for the Rayleigh distribution are established. The proposed $\mathrm{Bi}$ Rayleigh model has been compared with bi-normal ROC model and it is found that Bi-Rayleigh ROC model is able to classify more accurately that bi-normal ROC model when sample size is small. The confidence interval for the parameter $\sigma$ in case of large sample size is proposed. The theoretical results proposed in this paper are supported by real life examples as well as simulated data sets.

\section{ACKNOWLEDGMENT}

We would like to thank the Reviewers and Editors of the journal for their valuable comments and suggestions for improving the research paper.

\section{REFERENCES}

[1] Zhou XH, Obuchowski NA and Mcclish DK, "Statistical Methods in Diagnostic Statistics and Applied Probability 111", CRC Press, Florida, 2002.

[2] Krzanowski WJ and Hand DJ, "ROC curves for continuous data", Monographs on Medicine, John Wiley and Sons, New York, 2009.

[3] Hanley JA and McNeil BJ, "The Meaning And Use Of The Area Under A Receiver Operating Characteristic (ROC) Curve", Radiology, 143, 1, 29-36, 1982.

[4] Hanley JA and McNeil BJ, "A Method of Comparing the Area Under ROC Curves”, Radiology, 14, 29-36, 1983.

[5] Swets JA., "Indices of discrimination or diagnostic accuracy; their ROCs and implied models" Psychol Bul, 99, 181-198, 1986.

[6] Huang Y., and Pepe, MS, "A parametric ROC model-based approach for evaluating the predictiveness of continuous markers in case-control studies", Biometics, 65, 4, 1133-1144, 2009.

[7] Pepe, MS, "The statistical evaluation of medical tests for classification and prediction" University press, Oxford, 2000.

[8] Swets, JA, "ROC analysis applied to the evaluation of medical imaging techniques" Invest Radiology, 14,109-121, 1979.

[9] Papoulis A, "Probability, Random Variables, and Stochastic Processes", 2nd ed New York, McGraw-Hill, 104-148, 1984.

[10] Pan X, Metz CE, "The proper "binormal" model: parametric receiver Operating Characteristic curve estimation with degenerate data, Acad Radiology, 4, 380-389, 1997.

[11] Zhenqiu, L. and Ming, T, "ROC-based utility function maximization for feature selection and classification with applications to high-dimensional protease data", Biometrics, 64, 4, 1155-1161, 2008.

[12] Kundu, D., Gupta, R.D.. "Generalized exponential distribution: Existing results and some recent developments", Journal of Statistical Planning and Inference, 136, 3130-3144, 2007.

[13] Lloyd, C. J., "Using smoothed receiver operating characteristic curves to summarize and compare diagnostic systems", Journal of the American Statistical Association, 93, 1356-1364, 1998.

[14] Martin Betinec , "Testing the difference of the ROC Curves in Biexponential model", Tatra Mountains Mathematical Publications, 39, 215-223, 2006.

[15] Hanley, JE, "The robustness of the binormal assumptions used in fitting ROC curves", Medical Decision Making, 8, 197-203, 1988.

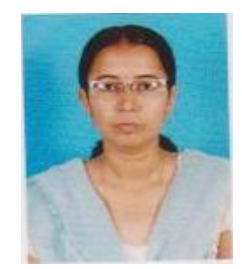

Dr. Sudesh Pundir is currently working as an Assistant Professor in the Department of Statistics, Pondicherry University, Puducherry. Her areas of research are Biostatistics, Applied Statistics, Reliability. She has a no. of published research papers in reputed journals. She has also participated in many conferences in India as well as abroad. She has organized one International Conference and acted as an organizing committee member in many conferences. She has presented many research papers and gave invited talks, special invited talks and acted as a resource person in various National and International
Conferences/Workshops. She is a life member of ISPS.

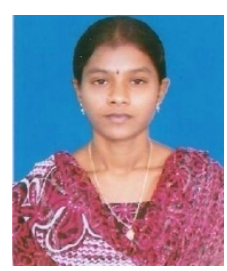

Amala. R did her M.Sc. Statistics with First class distinction at Department of Statistics, Ramanujan School of Mathematical Sciences, Pondicherry University, R.V. Nagar, Kalapet, Puducherry-605 014 during 2009-2011. She is presently pursuing her Ph.D. in Statistics under the guidance of Dr. Sudesh Pundir, Department of Statistics, Pondicherry University, Puducherry. Thrust area of her research is Applied Statistics. She has presented two research papers, one in an International Conference on Computational Statistics and Bio-Sciences at Pondicherry University and the other in a National Conference of National Symposium on Statistics and its Application for Young Researcher at Madras University Chennai. She has participated in 3 National conferences, one international conference and two Seminars. She has published one research paper in an international Journal. 